\title{
Erratum
}

\section{A Predator Prey Model with Age Structure}

J. M. Cushing and M. Saleem

J. Math. Biology (1982) 14: $231-250$

In addition to (2.3), $\beta(a)$ must satisfy $\int_{0}^{x} a \beta(a) e^{-\mu a} d a<+\infty$. To Theorem 2 should be added the phrase "provided that $J \geqslant 0$ when $h_{P}\left(R^{0}, P^{0}\right)<0$." and to the last sentence of its proof on the bottom of page 248 should be added the phrase "when $J=0$ at $\left(R^{0}, P^{0}\right)$, because then $\operatorname{Re} \zeta(m)=0(m) . "$. 